\title{
Application of Tactile Slippage Sensation Algorithm in Robot Hand Control System
}

\author{
${ }^{1}$ Hanafiah Yussof, ${ }^{1}$ Ahmed Jaffar, ${ }^{2}$ Nur Ismarrubie Zahari, ${ }^{3}$ Masahiro Ohka \\ ${ }^{1}$ Faculty of Mechanical Engineering \\ Universiti Teknologi MARA \\ 40450 Shah Alam, Malaysia \\ hanafi3010@hotmail, hanafiah1034@salam.uitm.edu.my \\ ${ }^{2}$ Department of Mechanical and Manufacturing Engineering \\ Faculty of Engineering, Universiti Putra Malaysia \\ 43400 Serdang, Selangor Malaysia \\ ${ }^{3}$ Graduate School of Information Science, Nagoya University \\ Furo-cho Chikusa-ku, Nagoya, Aichi, Japan
}

\begin{abstract}
This paper presents application of a new tactile slippage sensation algorithm in robot hand control system. The optical three-axis tactile sensor is a type of tactile sensor capable of defining normal and shear forces simultaneously. The tactile sensor is mounted on fingertip of robotic hand. Shear force distribution is used to define slippage sensation in the robot hand system. Based on tactile slippage analysis, a new control algorithm was proposed. To improve performance during object handling motions, analysis of slippage direction is conducted. The control algorithm is classified into two phases: grasp-move-release and grasptwist motions. Detailed explanations of the control algorithm based on the existing robot arm control system are presented. The experiment is conducted using a bottle cap, and the results reveal good performance of the proposed control algorithm to accomplish the proposed object handling motions.
\end{abstract}

Keywords: Tactile Sensor, Slippage Sensation Algorithm, Robot Hand, Ohject Handling.

\section{Introduction}

Sensorization of robotic hand is a big challenge in advancing grasping capability of robotic system. Tactile sensor is a type of sensing device that has great potential to enhance the robot grasping and handling capability [1][2]. The development of high performance tactile sensing device has becoming inevitably important issue in robotic field [3][4]. This paper presents the development of tactile sensing device and its control algorithm for application in robotic hands to archive human-like sensing ability.

The rationale for this endeavor was multifold. First, the growing interest in and rapid development of intelligent robotic hands such as the Gifu Hand III [5] and the high-speed robot hand [6] produced at the University of Tokyo, which perform impressive acts of dexterity and skillful manipulation, have urged the development of reliable tactile sensing devices and control algorithms to merge perfectly with the robot controller. In addition, such a sensing system can be used as an experimental apparatus to explore control strategies in dexterous robot manipulation [7] and may find a place in the rehabilitation of hand amputees, and replace humans in dangerous, dirty or time-consuming tasks.

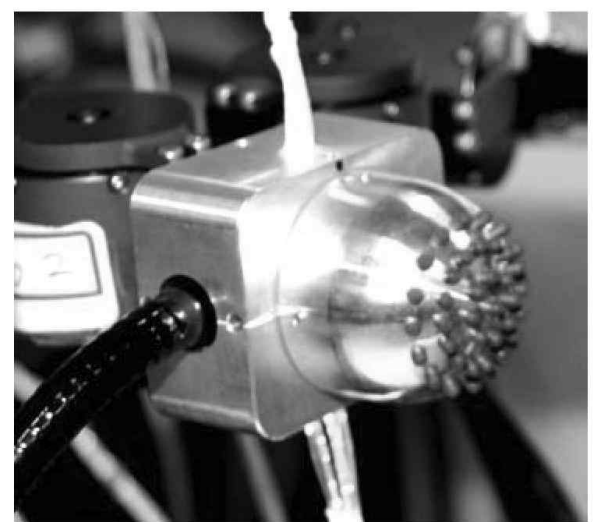

Fig. 1. Optical three-axis tactile sensors developed based on optical waveguide transduction method.

Tactile sensors offer great potential for improving the grasp synthesis in robot manipulation due to their extreme sensitivity and capability for measuring contact forces distribution [8] [9]. In previous work, the authors produced a unique tactile sensor system called optical three-axis tactile sensor for application to humanoid robot hands [10][11], as shown in Fig. 1. The sensing principle is based on the optical waveguide transduction method. 


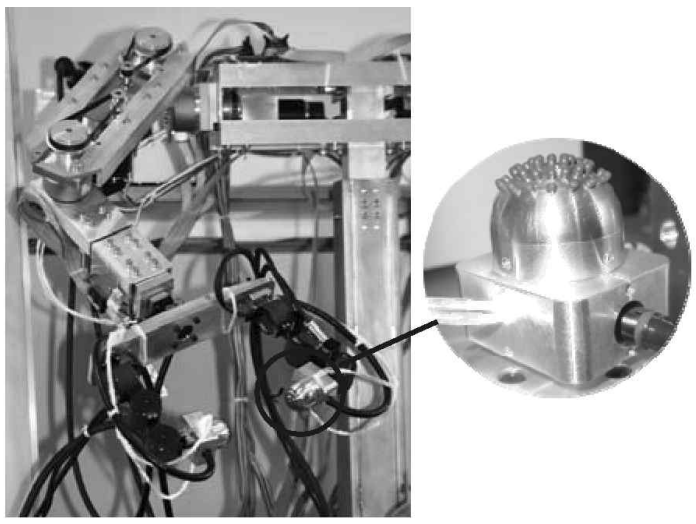

Fig. 2. An 11-dofs robot arm mounted with optical threeaxis tactile sensors.

The optical waveguide sensing principle used in this tactile sensor comparatively provides better sensing accuracy to detect contact phenomena from acquisition of three axial directions of forces [12]. Therefore, it is capable of measuring both normal and shear force simultaneously and suitable for application in robotic hands. For development and analysis of the control algorithm, the optical three axis tactile sensors are mounted on fingertips of a humanoid robot arm as shown in Fig. 2. The robot arm is consists of 11-dofs: 2-dofs at the shoulder joint, 1-dof at the elbow joint, 2-dofs at the wrist joint, and 2 units of 3-dof robot hands functioning as fingers for the arm.

In previous research, we have analyzed the active tactile sensing data in the robot arm control system to conduct a simple object handling tasks [13]. Furthermore, we analyzed grasp synthesis from distribution of normal and shear force to compile a control algorithm based on the tactile sensation. The analysis results were evaluated in an experiment of robot hand grasp and transfer of an object located at an arbitrary position [14]. From these, we realized that not only the tactile sensing is required to generate optimum grasp, but the slippage sensation is also important to support the control of grasp pressure for improvement of grasp quality during object manipulation.

In current research, we conduct further investigation to clarify the performance of the object manipulation task when tactile and slippage sensations were both integrated in the robot control system. To optimize the investigation, we create an experimental case study for the robot arm to complete and then we analyze the tactile slippage data. About the experimental task, humans commonly use their hands for a range of behaviors including power grips, fine manipulation, and communicative gestures. In order to closely describe these human behaviors in robotic experiments, we choose grasp, move and twist motions to evaluate our system. In this experiment the high accuracy of shear force detection of our tactile sensor system has provided a great opportunity to evaluate the slippage sensation of the robot hands.

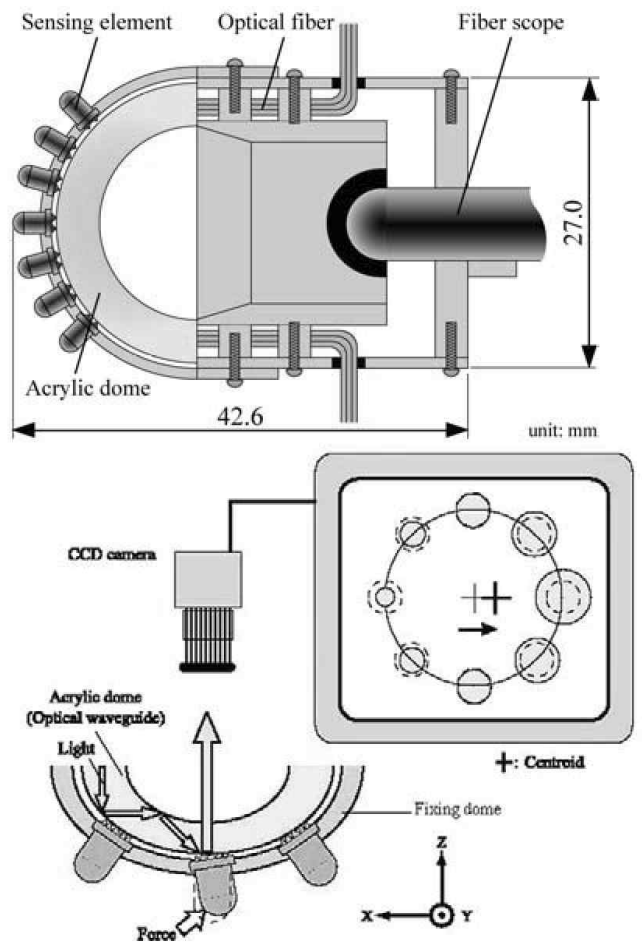

Fig. 3. Hardware structure of optical three-axis tactile sensor and sensing principle of shear force detection.

\section{Formatting instructions}

The hardware structure of the optical three-axis tactile sensor, as shown in Fig. 3, consists of an acrylic hemispherical dome, an array of 41 pieces of sensing elements made from silicon rubber, a light source, an optical fiber scope, and a CCD camera. The optical fiberscope is connected to the CCD camera to acquire the images of sensing elements touching the acrylic dome inside the sensor.

The silicone rubber sensing element is comprised of one columnar feeler and eight conical feelers that remain in contact with the acrylic surface. The light emitted from the light source is directed towards the edge of the hemispherical acrylic dome through optical fibers. When an object contacts the columnar feelers, resulting in contact pressure, the feelers collapse. At the points where the conical feelers collapse, light is diffusely reflected out of the reverse surface of the acrylic surface because the rubber has a higher reflective index. The contact phenomena, which consist of bright spots caused by the collapse of the feelers, are observed as image data, retrieved by the optical fiber scope connected to the CCD camera, and transmitted to the computer. In the measurement process, the normal force of the $F_{x}, F_{y}$ and $F_{z}$ values is calculated using integrated gray-scale value $G$, while shearing force is based on horizontal center point displacement. 


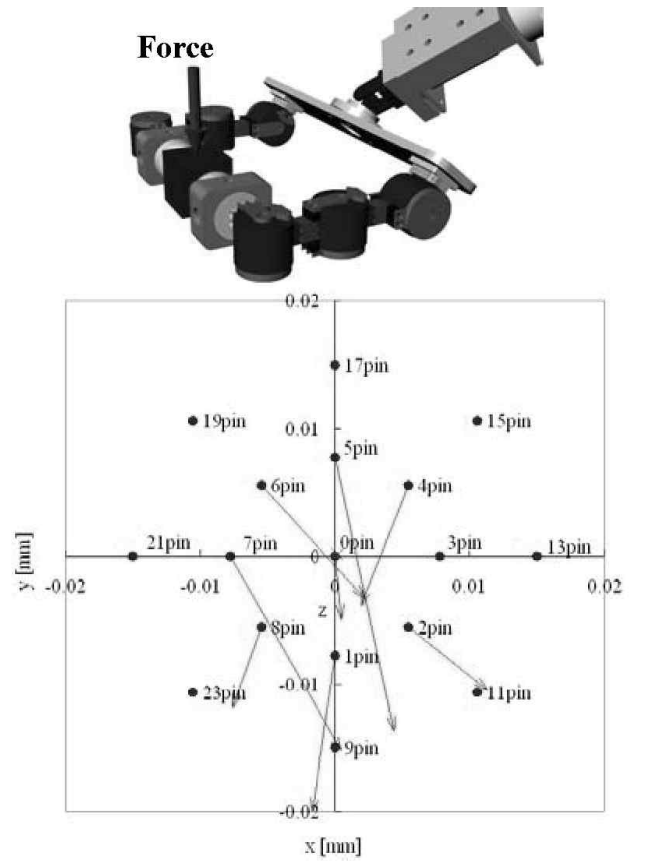

Fig. 4. Centroid displacement of tactile sensor elements in analysis of shear force and slippage direction when force is applied at object's center.

The displacement of gray-scale distribution $\boldsymbol{u}$ is defined in equation (1), where $\boldsymbol{i}$ and $\boldsymbol{j}$ are the orthogonal base vectors of the $x$ - and $y$-axes of a Cartesian coordinate, respectively. This equation is based on calibration experiments, and material functions are identified with piecewise approximate curves. Finally, each force component is defined in equation (2).

$$
\begin{gathered}
\boldsymbol{u}=u_{x} \boldsymbol{i}+u_{y} \boldsymbol{j} \\
F_{x}=f\left(u_{x}\right), F_{y}=f\left(u_{y}\right), F_{z}=g(G)
\end{gathered}
$$

\subsection{Methodology of Slippage Measurement}

In the shearing force detection, basically when the tangential force is applied to the sensing element, it collapses according to the applied load direction. At the same time, the centroid point of the bright spot is also shifted. Therefore, the shearing force can be calculated based on the horizontal displacement of this centroid point

In this study, due to the cylindrical shape of the sensing elements, slippage normally occurs when the grasped object surface alternates between sticking to the sensing element and sliding over the sensing element. In our system, this 'stick-slip' phenomenon can be measured by calculation of time derivation of centroid point displacement in shear force detection.

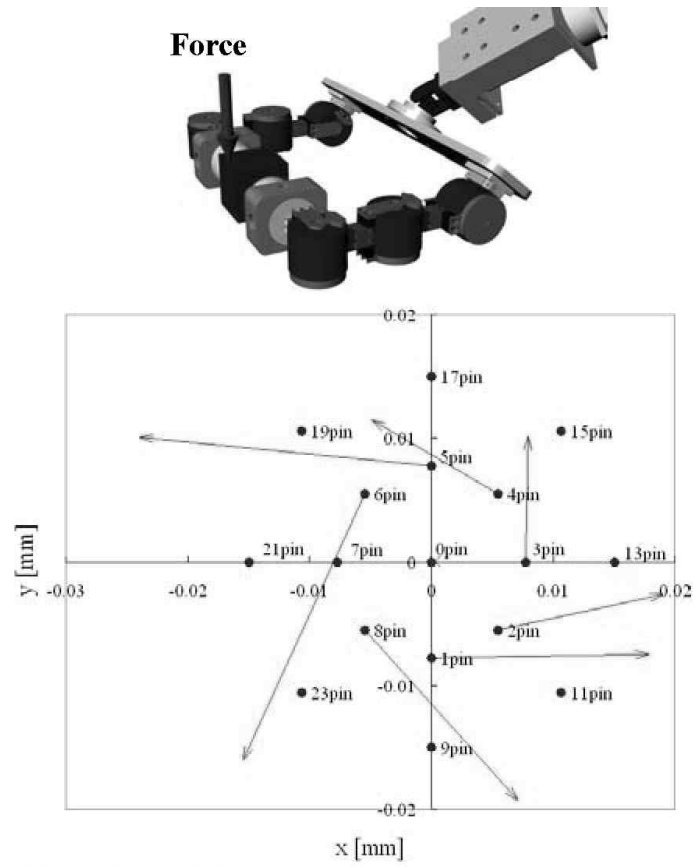

Fig. 5. Centroid displacement of tactile sensor elements in analysis of shear force and slippage direction when force is applied at object's edge.

Since the present control algorithm allows robot hands to refine their grasp pressure when slippage is detected, the robot hands will be able to handle various types of objects by adjusting the parameter value of the centroid point displacement threshold. It also useful to classify stiffness of an object for safe and effective manipulation [15]

The calculation of the centroid position, which is measured based on the center point of the bright spot area that equals the center position of the integrated gray-scale measurement area, is conducted when tangential force is applied to the sensor element or when slippage occurs. At this moment, the conical feeler's contact area at the sensing element with the acryl surface is shifted horizontally. To define the shearing force, we measure the horizontal centroid point displacement at the $x$ and $y$ axes.

In shear force measurement, by applying the increment of integrated gray-scale value $\Delta g(x, y)$, the centroid positions at the $x y$-axes, which are described as $x_{G}$ and $y_{G}$ are defined within the measurement area of the integrated gray-scale value, as shown in Eqs. (3) and (4), respectively:

$$
\begin{gathered}
x_{G}=\frac{\int_{S} \Delta g(x, y) x d S}{\int_{S} \Delta g(x, y) d S} \\
y_{G}=\frac{\int_{S} \Delta g(x, y) y d S}{\int_{S} \Delta g(x, y) d S} .
\end{gathered}
$$




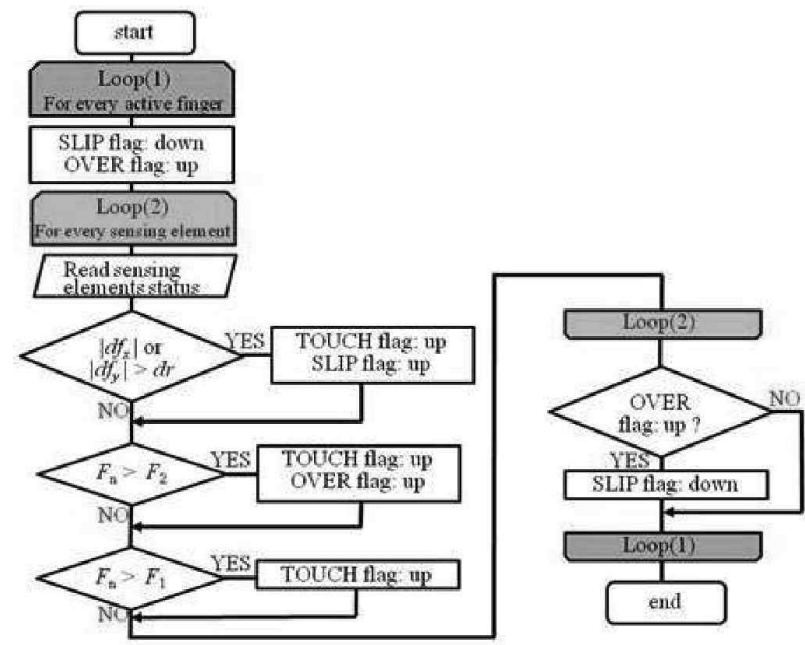

Fig. 6. Algorithm of flag analyzer.

Based on the above equations, the displacement of the centroid point at the $x y$-axes in time $t$ is defined as follows:

$$
\begin{aligned}
& d x_{G}^{(t)}=x_{G}^{(t)}-x_{G}^{(t-1)} \\
& d y_{G}^{(t)}=y_{G}^{(t)}-y_{G}^{(t-1)}
\end{aligned}
$$

\subsection{Analysis of Slippage Direction}

In object manipulation, when the robot arm is in the static condition and only the robot hand is performing motion, slippage normally occurs during the incipient grasp and when the object is in contact with the ground or another object surface. During the incipient grasp, the main reason for slippage is the object's surface condition and weight. However, it is possible to manipulate this condition for improvement of the task control. In order to find a suitable solution, we analyze the slippage direction of the optical three-axis tactile sensor.

In this study, we intended to use the slippage direction so that the robot system could achieve the autonomous grasping condition and define accurate timing to lift and release the object. In order to analyze slippage direction, we conducted a simple experiment in which the robot finger held a cube-shaped object as illustrated in Figs. 4 and 5. First, we applied force to the center of the object, as shown in Fig. 4, and then at its edge as shown in Fig. 5, which caused the object to slightly rotate. We defined the shear force characteristics and distribution by calculating the centroid point displacement of the sensing elements, as indicated in the graphs for both experiments.

The graphs in Figs. 4 and 5 show that the directions of the centroid displacements of the related tactile sensor elements are toward the applied force direction. In Fig. 5, the directions of the centroid displacement for the tactile sensor elements are according to the object's rotation direction. From both experiment results, we conclude that the shear force direction is according to the applied force direction. Therefore, slippage direction can be used as a control parameter in the robot arm control system to distinguish incipient grasp and release motion.

\section{Tactile Slippage Control Algorithm}

The objective of this paper is to evaluate the application of slippage control in the present robot arm control algorithm based on the tri-axial tactile sensing data In order to accomplish the objective of the present case study, we classified the control algorithm into two phases. Phase one is for grasp, move and release motions. Phase two is for grasp and twist motions during the twisting task.

The control system structure of the robot hand controller comprised of three modules: a Connection Module, Thinking Routines, and a Hand Control Module. The most important part is the Thinking Routines in which calculation of tri-axial tactile data and decision of velocity and motion control are conducted.

In the present robot hand control system, the robot hand is controlled according to velocity control. Meanwhile, the hand motion is divided into two modes. First is "search mode" to make fingers approach an object. After fingers touch the object, the hand status enters "move mode" to manipulate the object. During both search and move modes, when absolute time derivative of shearing force of a sensing element exceeds a threshold $d r$, this system achieved the condition as slippage. Furthermore, to prevent the hands from dropping the object due to the slippage, re-compressive velocity is defined to move the hands along counter direction of applied force.

In addition, to define optimum grasp pressure toward the object, we fixed two layers of normal force threshold $F_{1}$ and $F_{2}$. When normal force exceeds $F_{1}$, the robot hands will stop the gripping motion and hold its position. $F_{2}$ is for emergency stop to prevent the robot hands from crushing the object or damaging the sensor elements.

In our system, the sensor control program and hand control program are executed in different computers because CPU time is efficiently consumed using the multitask program method. These programs are synchronized as the following five flags according to the tri-axial tactile data and finger motions:

SEARCH: Hands search for an object until normal force of a sensing element exceeds a threshold $F_{1}$ or a Slip flag is raised.

MOVE: This flag is raised whenever the robotic hand manipulates an object.

TOUCH: This flag is raised whenever one of the fingers touches an object.

SLIP: $\quad$ This flag is raised whenever time derivative of shearing force exceeds a threshold $d r$.

OVER: This flag is raised when normal force of a sensing element exceeds a threshold $F_{2}$. 
Fig. 6 shows the algorithm of the flag analyzer in the control system. The outputs of this algorithm are TOUCH, SLIP and OVER flags. In the flag analyzer, the control system realizes the status as touching an object when normal force of a sensing element exceeds $F_{1}$ or absolute time derivative of shearing force exceeds $d r$ (SLIP flag is raised). At this moment, the TOUCH flag is raised. The OVER flag is raised when normal force of a sensing element exceeds $F_{2}$ to prevent oversqueezing toward the object.

Fig. 7 shows the algorithm of the velocity generator inside the Thinking Routines. The velocity of the finger tip is determined based on the five flag values. Whenever the SLIP flag is raised, a sensing element of the largest normal force is identified and re-compressive velocity of the finger is determined as an inward normal line of the sensing element. The re-compressive velocity is added to the current velocity, and the resultant velocity is applied to the control module.

\subsection{Phase I: Grasp-Move-Release}

The first phase of the control algorithm is for grasp, move and release motions. Initially, based on the slippage direction analysis presented in the previous section, when the direction of centroid displacement is at the positive side, the control system will classify the condition as a grasp motion. If the direction is at the negative side, the system will classify it as a release motion.

During grasping motion, when the fingers have defined a suitable grasp pressure on an object, the finger controller sends a 'Grasping Ended' instruction to the arm controller so that the arm can start to lift and move the object. At this time, when slippage caused by the movement of the arm or the object's weight is detected, the finger controls the grip by re-compressing the object so that the object will not drop. Next, the arm moves to the targeted position while maintaining its orientation holding the object. Then the arm controller sends an instruction to the finger controller to start the process of releasing the object. At this time, both hands move to the lower position toward the ground. Finally, the object comes into contact with the ground where slippage is detected due to the object contacting the ground.

At this time, the hand controller chooses only one sensor element that shows the maximum normal force value among sensor elements where slippage was detected. This sensor element is used as a coordinate point to define the direction of slippage. The reason for this selection method is that the object seems to rotate when it comes into contact with the ground, and the sensor pin that has shown the maximum normal force value has become the center point of this rotation. After the direction of slippage is confirmed, the robot hands will gently release the object.

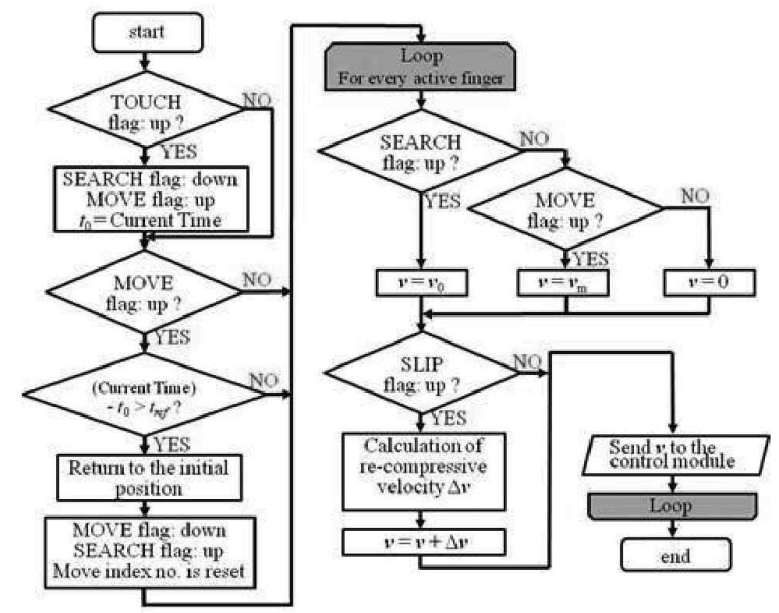

Fig. 7. Algorithm of finger speed estimator.

\subsection{Phase II: Grasp-Twist}

The second phase is for grasp and twist motions during the twisting task. Since turning motion is different from grasp and release motion, the control algorithm of slippage direction is not considered in this motion control. We only considered the increment of time derivative of shearing force together with increment of normal force. Hence, the control parameters involved are $d r, F_{1}$ and $F_{2}$. Furthermore, since the twisting cap problem requires touch and release motion, MOVE and SEARCH flags are controlled according to TOUCH and SLIP flags and spending time. A motion trajectory was generated for both hands to perform grasping and twisting motions. At first, robot fingers grasp the object, move in the turning direction, and return back again to the initial grasping position to repeat the twisting task.

The grasp pressure is controlled by the aforementioned parameters. During twisting motion, the important point is to decide how and when to finish the motion [16]. During closing the bottle cap, two control conditions were identified. First is when the detected data of displacement in the shear force detection (slippage detection) exceeds the threshold $d r$ at specified times (Ns) in one cycle of the twisting task.

Second is when the slippage detection exceeds the threshold $d r$ in two continuous cycles of the twisting task. The robot control system will realize the cap is fully tightened when either one of the above conditions is satisfied. The robot hand can stop the task when the cap is fully tightened. 


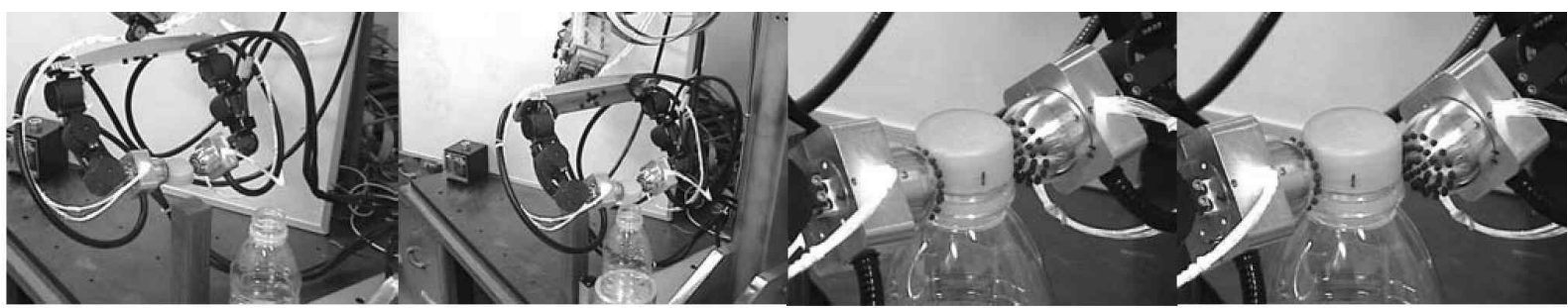

Fig.8. Sequential photographs in the experiment using humanoid robot arm with optical three-axis tactile sensor.

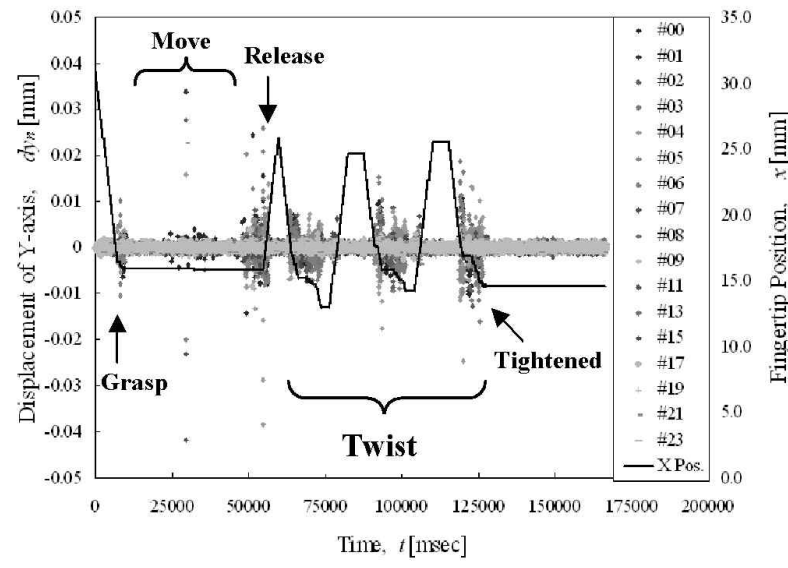

Fig. 9. Relationship between displacement of $y$-directional centroid change and $x$-directional fingertip position.

\section{Experiment and Result}

Experiment of grasp, move and twist object using the humanoid robot arm equipped with a tactile sensor was conducted to evaluate the performance of the proposed tactile slippage control algorithm. The object is a mineral water bottle cap. In this experiment, the cap and bottle are located at the place where only the geometrical positions of the $x$ - and $y$-axes are primarily defined. The height at the $z$-axis is not specified. This is to evaluate the proposed slippage direction control in grasp and release motions. In the twisting task, the robot hand will use the reference coordinate during release motion.

At this time, $N s$ value is fixed at 8 times based on calibration. The $d r$ value is 0.004 [mm] for the whole tasks except for twisting task which was increased 1.3 times so that the tactile sensor can optimize slippage detection to justify the cap tighten condition. Fig. 8 shows pictures of the experimental condition. First, the robot hands grasp the object and adjust its grasp pressure. At this moment, since the first detected shear force direction that exceeded the $d r$ value is at the positive side, the robot arm continues lifting the object and moves to the bottle position. During the move motion, slippage was detected probably due to vibration of the arm. At this moment, robot hands re-compress the cap to keep it from dropping.
When the robot hand moves toward the bottle, the cap touched the bottle head surface causing great slippage due to rotation of the cap. Since the first detected slippage direction that exceeded $d r$ value is at the negative side, the robot hands gently release the cap. Next, the robot hands performed twisting tasks.

At the third cycle of the tasks, the robot controller realized that the cap was already tightened because the slippage detection exceeded the fixed $N_{S}$ value. The robot arm managed to perform all motions smoothly. The performance of tactile slippage sensation in this robot system was verified in graph of relationship between shear force displacement data at the $y$-axis and the fingertip position of the robot hand at the $x$-axis, as shown in Fig. 9 .

\section{Conclusions}

In this report, we proposed a new control algorithm in a robot arm based on tactile slippage sensation. We used the optical three-axis tactile sensor mounted on fingertips of the robot hands. The proposed algorithm was evaluated in an experimental case study of grasp, move and twisting motions. Prior to the experiment, we presented the methodology of slippage measurement and conducted analysis of slippage direction to improve performance of the proposed algorithm.

To satisfy the proposed case study, the algorithm was classified in two phases, grasp-move-release and grasptwist. The experimental results using a bottle cap revealed good performance of the proposed algorithm for future application in a real artificial robot hand. This result shows that integration of tactile and slippage sensation in a robot control system could contribute a better maneuvering of the robot arm-finger system when handling an object, particularly when the geometrical orientation of the object is unknown.

\section{Acknowledgements}

The authors would like to express their appreciation to the Ministry of Higher Education Malaysia (MOHE), Universiti Teknologi MARA (UiTM), University Putra Malaysia and Nagoya University, Japan for their support. 


\section{References}

[1] D. Göger, N. Gorges and H. Wörrn, Tactile Sensing for an Anthropomorphic Robotic Hand: Hardware and Signal Processing, 2009 IEEE Int. Conf. on Robotics and Automation, pp. 895-901, 2009.

[2] Y. Hasegawa, M. Shikida, T. Shimizu, T. Miyaji, H. Sakai, $\mathrm{K}$. Sato and K. Itoigawa, A Micromachined Active Tactile Sensor for Hardness Detection, Sensors and Actuators (A Physical), Vol. 114(2-3), pp. 141-146, 2004.

[3] V. A. Ho, D. V. Dao, S. Sugiyama and S. Hirai, (). Analysis of Sliding of a Soft Fingertip Embedded with a Novel Micro Force/Moment Sensor: Simulation, Experiment, and Application. 2009 IEEE Int. Conf. on Robotics and Automation, pp. 889-894, 2009.

[4] H. Maekawa, K. Tanie, K. Komoriya, M. Kaneko, C. Horiguchi and T. Sugawara, Development of a Fingershaped Tactile Sensor and Its Evaluation by Active Touch. 1992 IEEE Int. Conf. on Robotics and Automation, pp. $1327-1334,1992$

[5] T. Mouri and H. Kawasaki, "Anthropomorphic Robot Hand: Gifu Hand III", in proceeding ICCAS2009, pp. 1288-1293, Korea, 2009.

[6] T. Senoo, Y. Yamakawa, S. Mizusawa, A. Namiki, M. Ishikawa, and M. Shimojo, "Skillful Manipulation Based on High-speed Sensory-Motor Fusion", in proc. ICRA2009, pp.1611-1612, Kobe, 2009.

[7] T. Mouri, H. Kawasaki and S. Ito, "Unknown Object Grasping Strategy Imitating Human Grasping Reflex for Anthropomorphic Robot Hand", Joumal of Advanced Mechanical Design, Systems, and Manufacturing, 1(1), pp. 1-11, JSME, 2007.

[8] H. R. Nicholls, Tactile Sensing Using an Optical Transduction Method, Traditional and Nontraditional Robot Sensors (Edited by T. C. Henderson), SpringerVerlag, pp. 83-99, 1990.
[9] H. R. Nicholls and M. H. Lee (). A Survey of Robot Tactile Sensing Technology. International Journal of Robotics Research, Vol 8(3), pp. 3-30, 1989.

[10] M. Ohka, Y. Mitsuya, Y. Matsunaga and S. Takeuchi, "Sensing Characteristics of an Optical Three-Axis Tactile Sensor Under Combined Loading", Robotica, vol.22, pp. 213-221, 2004.

[11] M. Ohka, H. Kobayashi and Y. Mitsuya, "Sensing Characteristic of an Optical Three-Axis Tactile Sensor Mounted on a Multi-Fingered Robotic Hand", in proc. IROS2005, pp. 1959-1964.

[12] H. Yussof, M. Ohka, H. Kobayashi, J. Takata, M. Yamano, Y. Nasu, "Development of an Optical Three-axis Tactile Sensor for Object Handling Tasks in Humanoid Robot Navigation System," Studies in Computational Intelligence, vol. 76, pp. 43-51, 2007.

[13] H. Yussof, J. Wada, M. Ohka, "Object Handling Tasks Based on Active Tactile and Slippage Sensation in a MultiFingered Humanoid Robot Arm," in proc. ICRA2009, pp. 502-507, Kobe, Japan, May 12-17, 2009

[14] H. Yussof, J. Wada, M. Ohka, "Grasp Synthesis Based on Tactile Sensation in Robot Manipulation of Arbitrary Located Object," in proc. AIM2009, pp. 560-565, Singapore, July 14-17, 2009.

[15] H. Yussof, M. Ohka, A. R. Omar, M. A. Ayub, "Determination of Object Stiffness Control Parameters in Robot Manipulation Using a Prototype Optical Three-Axis Tactile Sensor," in proc. SENSORS2008, pp. 992-995, Lecce, Italy, Oct. 26-29, 2008.

[16] C. Xiong, Y. Li, Y. Xiong, H. Ding, "Grasp Capability Analysis of Multifingered Robot Hands", J. Robotics and Autonomous Systems, vol. 27, pp. 211-224, 1999. 